\title{
Application of Two-Dimensional Compressive Sensing to Wavelet Method of Moments for Fast Analysis of Wide-Angle Electromagnetic Scattering Problems
}

\author{
Yi Liu, ${ }^{1,2,3}$ Qi Qi $\mathbb{C}^{3}{ }^{3}$ Xinyuan Cao, ${ }^{3}$ Mingsheng Chen, ${ }^{3}$ Guoqing Deng, ${ }^{1,2}$ Zhixiang Huang, \\ and Xianliang $\mathrm{Wu}^{4}$ \\ ${ }^{1}$ Anhui Institute of Optics and Fine Mechanics, Hefei Institutes of Physical Science, Chinese Academy of Sciences, \\ Hefei 230031, China \\ ${ }^{2}$ University of Science and Technology of China, Hefei 230026, China \\ ${ }^{3}$ Anhui Province Key Laboratory of Simulation and Design for Electronic Information System, Hefei Normal University, \\ Hefei 230061, China \\ ${ }^{4}$ Key Laboratory of Intelligent Computing \& Signal Processing, Ministry of Education, Anhui University, Hefei 230601, China \\ Correspondence should be addressed to Qi Qi; microwaveqq@126.com
}

Received 5 March 2021; Revised 27 July 2021; Accepted 10 August 2021; Published 20 August 2021

Academic Editor: Paolo Baccarelli

Copyright ( 2021 Yi Liu et al. This is an open access article distributed under the Creative Commons Attribution License, which permits unrestricted use, distribution, and reproduction in any medium, provided the original work is properly cited.

\begin{abstract}
To efficiently solve the electromagnetic scattering problems over a wide incident angle, a novel scheme by introducing the twodimensional compressive sensing theory into the wavelet method of moments is proposed. In this scheme, a linear system of equations with multiple right-hand sides in wavelet domain is formed firstly, and one side of the bilateral sparse transform to the induced current matrix is simultaneously accomplished and then the bilateral measurement of the induced current matrix is operated by the linear superposition of the right-hand side vectors a few times and the extraction of rows from the impedance matrix. Finally, after completing the other side of the bilateral sparse transform, the wide-angle problems can be solved rapidly by two times of recovery algorithm with prior knowledge. The basic principle is elaborated in detail, and the effectiveness is demonstrated by numerical experiments.
\end{abstract}

\section{Introduction}

Method of moments (MoM) [1] is an accurate and efficient method for solving electromagnetic scattering problems. Many fast methods based on MoM have been proposed, such as fast multipole method (FFM) [2], adaptive integral method (AIM) [3], adaptive cross approximation (ACA) [4], and wavelet MoM [5]. However, unless the approximated technique (e.g., asymptotic waveform evaluation [6]) is applied, the traditional MoM needs to be implemented repeatedly at every incident angle increment for solving the wide-angle electromagnetic scattering problems, which leads to a huge computing amount.

Recently, a theory known as compressive sensing (CS) [7] is put forward in the field of signal processing, by which the limitation from the Nyquist sampling theorem can be broken and has been successfully applied to the computation electromagnetic [8-11]. By combining the CS theory and the traditional MoM, a scheme for the rapid analysis of wideangle problems has been formed [12]. In this scheme, a new source including much information from different incident angles is constructed, and the measurement of induced currents is obtained by multiple calculations of the traditional MoM under the new source, and then the original induced currents over the wide angle can be approximated by means of the sparse transform and recovery algorithm.

In this paper, the two-dimensional (2-D) CS theory [13] is employed in the wavelet MoM to build a more efficient scheme for wide-angle problems, in which the bilateral measurement and the bilateral sparse transform are used to 
the induced currents and the reconstruction of induced currents is operated by the twice recovery algorithm. The specific formulas are deduced in detail, and numerical examples of differently shaped objects are presented.

\section{Formulations}

2.1. Conventional CS Scheme. The matrix equation of traditional MoM for solving the wide-angle electromagnetic scattering problems can be written as

$$
\mathbf{Z}\left[\mathbf{I}_{1} \mathbf{I}_{2}, \cdots, \mathbf{I}_{n}\right]=\left[\mathbf{V}_{1} \mathbf{V}_{2}, \cdots, \mathbf{V}_{n}\right],
$$

where $\mathbf{Z}$ is the impedance matrix, $\mathbf{I}_{1}$ to $\mathbf{I}_{n}$ and $\mathbf{V}_{1}$ to $\mathbf{V}_{n}$ represent the $n$ induced current vectors and the $n$ corresponding excitation vectors at $n$ different incident angles, respectively.

The conventional CS scheme in [12] mainly consists of the following three steps:

Step 1. $M$ new incident sources based on CS theory are formed as

$$
\mathbf{V}_{i}^{\mathrm{CS}}=c_{i 1} \mathbf{V}_{1}+c_{i 2} \mathbf{V}_{2}+, \cdots,+c_{i n} \mathbf{V}_{n}, \quad(i=1,2, \cdots, M),
$$

in which $c_{i j}$ is the random coefficient.

Step 2. By using the new sources, $M$ corresponding current vectors based on CS theory can be obtained by

$$
\mathbf{Z}\left[\mathbf{I}_{1}^{\mathrm{CS}} \mathbf{I}_{2}^{\mathrm{CS}}, \cdots, \mathbf{I}_{M}^{\mathrm{CS}}\right]=\left[\mathbf{V}_{1}^{\mathrm{CS}} \mathbf{V}_{2}^{\mathrm{CS}}, \cdots, \mathbf{V}_{M}^{\mathrm{CS}}\right]
$$

Considering the linear identity of the problem, the current vectors can be represented as

$$
\mathbf{I}_{i}^{\mathrm{CS}}=c_{i 1} \mathbf{I}_{1}+c_{i 2} \mathbf{I}_{2}+, \cdots,+c_{i n} \mathbf{I}_{n}, \quad(i=1,2, \cdots, M) .
$$

In the view of CS theory, $\mathbf{I}_{1}^{\mathrm{CS}}$ to $\mathbf{I}_{M}^{\mathrm{CS}}$ can be regarded as the results of $M$ measurements of $\left[\mathbf{I}_{1} \mathbf{I}_{2}, \cdots, \mathbf{I}_{n}\right]$.

Step 3. With the help of the sparse transform (e.g., fast Fourier transform (FFT)), one can obtain

$$
\left[\begin{array}{cccc}
c_{11} & c_{12} & \cdots & c_{1 n} \\
\vdots & \vdots & \cdots & \vdots \\
c_{M 1} & c_{M 2} & \cdots & c_{M n}
\end{array}\right]\left[\mathbf{I}_{1} \mathbf{I}_{2}, \cdots, \mathbf{I}_{n}\right]^{\mathrm{T}}=\boldsymbol{\Phi} \Psi\left[\boldsymbol{\alpha}_{1} \boldsymbol{\alpha}_{2}, \cdots, \boldsymbol{\alpha}_{N}\right]=\left[\mathbf{I}_{1}^{\mathrm{CS}} \mathbf{I}_{2}^{\mathrm{CS}}, \cdots, \mathbf{I}_{M}^{\mathrm{CS}}\right]^{\mathrm{T}}
$$

in which $\left[c_{i j}\right]$ is the measurement matrix denoted as $\Phi$, $\boldsymbol{\alpha}_{1}$ to $\boldsymbol{\alpha}_{N}$ are the sparse projections of each column of $\left[\mathbf{I}_{1}\right.$ $\left.\mathbf{I}_{2}, \cdots, \mathbf{I}_{n}\right]^{\mathrm{T}}$ in the sparse transform $\boldsymbol{\Psi}$, and $N$ is the number of basis functions.

$$
\left[\widehat{\boldsymbol{\alpha}}_{1} \widehat{\boldsymbol{\alpha}}_{2}, \cdots, \widehat{\boldsymbol{\alpha}}_{N}\right]=\operatorname{argmin}\left\|\left[\widehat{\alpha}_{1} \widehat{\alpha}_{2}, \cdots, \widehat{\alpha}_{N}\right]\right\|_{L} \text { s.t. }(\boldsymbol{\Phi} \Psi)\left[\boldsymbol{\alpha}_{1} \boldsymbol{\alpha}_{2}, \cdots, \boldsymbol{\alpha}_{N}\right]=\left[\mathbf{I}_{1}^{\mathrm{CS}} \mathbf{I}_{2}^{\mathrm{CS}}, \cdots, \mathbf{I}_{M}^{\mathrm{CS}}\right]^{\mathrm{T}}
$$

and the original induced currents are reconstructed by

$$
\mathbf{I}_{i}^{\mathrm{CS}}=c_{i 1} \mathbf{I}_{1}+c_{i 2} \mathbf{I}_{2}+, \cdots,+c_{i n} \mathbf{I}_{n}, \quad(\mathbf{I}=1,2, \cdots, M) .
$$

2.2. 2-D CS Scheme Based on Wavelet MoM. First, by the wavelet transform, (1) is transformed into

$$
\widetilde{\mathbf{Z}}\left[\widetilde{\mathbf{I}}_{1} \widetilde{\mathbf{I}}_{2}, \cdots, \widetilde{\mathbf{I}}_{n}\right]=\left[\widetilde{\mathbf{V}}_{1} \widetilde{\mathbf{V}}_{2}, \cdots, \widetilde{\mathbf{V}}_{n}\right],
$$

where $\widetilde{\mathbf{Z}}=\mathbf{W Z W} \mathbf{W}^{\mathrm{T}}, \quad\left[\widetilde{\mathbf{I}}_{1} \widetilde{\mathbf{I}}_{2}, \cdots, \widetilde{\mathbf{I}}_{n}\right]=\mathbf{W}\left[\mathbf{I}_{1} \mathbf{I}_{2}, \cdots, \mathbf{I}_{n}\right], \quad\left[\widetilde{\mathbf{V}}_{1}\right.$ $\left.\widetilde{\mathbf{V}}_{2}, \cdots, \widetilde{\mathbf{V}}_{n}\right]=\mathbf{W}\left[\mathbf{V}_{1} \mathbf{V}_{2}, \cdots, \mathbf{V}_{n}\right]$, and $\mathbf{W}$ is an orthogonal matrix constructed by standard fast wavelet transform (FWT) with Symlets wavelet $[15,16]$. In the wavelet domain, the sparse impedance matrix and the sparse excitation matrix are obtained after thresholding, and one side of the bilateral sparse transform to the induced currents is accomplished.

Second, since the impedance matrix is indirectly coincident with the restricted isometry property [17], an
Step 4. With the utilization of the recovery algorithm (e.g., orthogonal matching pursuit (OMP) [14]), the projections can be approximated by underdetermined system of equations can be established [18] as

$$
\widetilde{\mathbf{Z}}_{p}\left[\widetilde{\mathbf{I}}_{1} \widetilde{\mathbf{I}}_{2}, \cdots, \widetilde{\mathbf{I}}_{n}\right]=\left[\widetilde{\mathbf{V}}_{1 p} \widetilde{\mathbf{V}}_{2 p}, \cdots, \widetilde{\mathbf{V}}_{n p}\right]
$$

in which $\left[\tilde{\mathbf{V}}_{1 p}, \widetilde{\mathbf{V}}_{2 p}, \cdots, \widetilde{\mathbf{V}}_{n p}\right]$ is composed of all $p$ nonzero rows of $\left[\tilde{\mathbf{V}}_{1} \tilde{\mathbf{V}}_{2}, \cdots, \tilde{\mathbf{V}}_{n}\right]$ and $\widetilde{\mathbf{Z}}_{p}$ is constructed by extracting the corresponding $p$ rows from $\widetilde{\mathbf{Z}}$. In the CS theory, $\widetilde{\mathbf{Z}}_{p}$ is considered as the measurement matrix to each column of $\left[\widetilde{\mathbf{I}}_{1} \widetilde{\mathbf{I}}_{2}, \cdots, \widetilde{\mathbf{I}}_{n}\right]$ and $\widetilde{\mathbf{V}}_{1 p}$ to $\widetilde{\mathbf{V}}_{n p}$ are the measurement results. Third, $M^{\prime}$ new excitation vectors with the length of $p$ based on CS theory are formed in the wavelet domain as

$$
\widetilde{\mathbf{V}}_{i p}^{\mathrm{CS}}=c_{i 1} \tilde{\mathbf{V}}_{1 p}+c_{i 2} \tilde{\mathbf{V}}_{2 p}+, \cdots,+c_{i n} \widetilde{\mathbf{V}}_{n p} \quad\left(i=1,2, \cdots M^{\prime}\right),
$$

and $M^{\prime}$ measurement results of $\left[\widetilde{\mathbf{I}}_{1} \widetilde{\mathbf{I}}_{2}, \ldots, \widetilde{\mathbf{I}}_{n}\right]$ are acquired by

$$
\widetilde{\mathbf{Z}}_{p}\left[\widetilde{\mathbf{I}}_{1}^{\mathrm{CS}} \widetilde{\mathbf{I}}_{2}^{\mathrm{CS}}, \cdots, \widetilde{\mathbf{I}}_{M^{\prime}}^{\mathrm{CS}}\right]=\left[\widetilde{\mathbf{V}}_{1 p}^{\mathrm{CS}} \widetilde{\mathbf{V}}_{2 p}^{\mathrm{CS}}, \cdots, \widetilde{\mathbf{V}}_{M^{\prime} p}^{\mathrm{CS}}\right] \text {. }
$$


Similarly to (4), we can rewrite (11) as

$$
\widetilde{\mathbf{Z}}_{p}\left[\widetilde{\mathbf{I}}_{1} \widetilde{\mathbf{I}}_{2}, \cdots, \widetilde{\mathbf{I}}_{n}\right] \Phi^{\mathrm{T}}=\left[\widetilde{\mathbf{V}}_{1 p}^{\mathrm{CS}} \widetilde{\mathbf{V}}_{2 p}^{\mathrm{CS}}, \cdots, \widetilde{\mathbf{V}}_{M^{\prime} p}^{\mathrm{CS}}\right],
$$

in which $\Phi^{\mathrm{T}}$ is the measurement matrix to the rows of $\left[\widetilde{\mathbf{I}}_{1} \widetilde{\mathbf{I}}_{2}, \cdots, \widetilde{\mathbf{I}}_{n}\right]$.

Afterwards, the other side of the bilateral sparse transform is applied as

$$
\left[\widetilde{\mathbf{I}}_{1} \widetilde{\mathbf{I}}_{2}, \cdots, \widetilde{\mathbf{I}}_{n}\right]=\Gamma \Psi,
$$

where $\Psi$ is the sparse transform matrix to the rows of $\left[\widetilde{\mathbf{I}}_{1} \widetilde{\mathbf{I}}_{2}, \cdots, \widetilde{\mathbf{I}}_{n}\right]$ and $\boldsymbol{\Gamma}$ represents the sparse projection. Substituting it into (12), one will get

$$
\widetilde{\mathbf{Z}}_{p} \Gamma \Psi \boldsymbol{\Phi}^{\mathrm{T}}=\left[\tilde{\mathbf{V}}_{1 p}^{\mathrm{CS}} \tilde{\mathbf{V}}_{2 p}^{\mathrm{CS}}, \cdots, \tilde{\mathbf{V}}_{M^{\prime} p}^{\mathrm{CS}}\right]
$$

Finally, the projection $\Gamma$ can be approximated by using the recovery algorithm to solve the following two optimization problems:

$$
\widehat{\boldsymbol{s}}=\arg \min \left\|\Gamma \Psi \boldsymbol{\Phi}^{\mathrm{T}}\right\|_{L} \text { s.t. }\left\|\widetilde{\mathbf{Z}}_{p}\left(\Gamma \Psi \boldsymbol{\Phi}^{\mathrm{T}}\right)-\left[\widetilde{\mathbf{V}}_{1 p}^{\mathrm{CS}} \widetilde{\mathbf{V}}_{2 p}^{\mathrm{CS}}, \cdots, \widetilde{\mathbf{V}}_{M^{\prime} p}^{\mathrm{CS}}\right]\right\|_{L}<\varepsilon,
$$

$$
\widehat{\Gamma}=\arg \min \|\Gamma\|_{L} \text { s.t. }\left\|\Gamma\left(\Psi \Phi^{T}\right)-\widehat{s}\right\|_{L}<\mathcal{\varepsilon},
$$

and the original induced currents can be reconstructed by

$$
\left[\widehat{\mathbf{I}}_{1} \widehat{\mathbf{I}}_{2}, \cdots, \widehat{\mathbf{I}}_{n}\right]=\mathbf{W}^{T} \widehat{\Gamma} \boldsymbol{\Psi} .
$$

Considering the prior knowledge that $\left[\widetilde{\mathbf{I}}_{1} \widetilde{\mathbf{I}}_{2}, \ldots, \widetilde{\mathbf{I}}_{n}\right]$ contains some zero rows, there are necessarily zero rows of the same amount and the same index in $\widehat{s}$, that is, the measurement result of the rows of $\left[\widetilde{\mathbf{I}}_{1} \widetilde{\mathbf{I}}_{2}, \cdots, \widetilde{\mathbf{I}}_{n}\right]$, and therefore, only the nonzero rows in the projection $\Gamma$, which are corresponding to the nonzero rows in $\widehat{s}$, need to be recovered in (16). Thus, considerable computing time can be saved.

In the proposed scheme, the solution of two optimization problems is dominating in the computing cost. The total computational complexity of solving (15) and (16) is $O\left(p N S_{1} M^{\prime}+n Q S_{2} M^{\prime}\right.$ ) (take OMP as the recovery algorithm), where $S_{1}$ and $S_{2}$ are the iteration steps in the two times of OMP, respectively, $n$ is the number of different incident angles, $N$ is the number of basis functions, $p$ is the number of rows extracted from the impedance matrix, and $Q$ is the number of nonzero rows in $\boldsymbol{\Gamma}$. The computational complexity of traditional MoM with iteration method for analyzing wide-angle problems is $O\left(n d N^{2}\right)$, where $d$ is the iteration counter.

Therefore, the effectiveness improved by the 2-D CS scheme can be evaluated by

$$
\zeta=\frac{p N S_{1} M^{\prime}+n Q S_{2} M^{\prime}}{n d N^{2}}=\frac{p S_{1} M^{\prime}}{n d N}+\frac{Q S_{2} M^{\prime}}{d N^{2}},
$$

in which $p<<N, Q<<N$, and $M^{\prime}<<n$, and in general, $S_{1}<<d, S_{2}<<d$, and $M^{\prime}<<N$.

Furthermore, the computational complexity of the conventional CS scheme provided in [12] is $O\left(d M N^{2}+n M K N\right)$, where $K$ is the iteration steps of OMP.
Compared with the conventional scheme, the proposed scheme also has the following two improvements: first, the calculation amount of matrix operation involving the impedance matrix is reduced since the impedance matrix in the wavelet domain is sparse; then, the number of measurements is decreased $\left(M^{\prime}<M\right)$ because the sparsity of the projection $\Gamma$ is better with the help of the bilateral sparse transform. Obviously, $p N S_{1} M^{\prime}<d M N^{2}$ and $n Q S_{2} M^{\prime}<n M K N$.

\section{Numerical Results}

In this section, four examples of differently shaped objects are presented to validate the effectiveness of the proposed scheme, in which the electric field integral equation (EFIE) is established to solve the problems; Gaussian matrix, FFT basis, and OMP are taken as the measurement matrix $\Phi$, the sparse transform matrix $\Psi$ and recovery algorithm, respectively, and the incident waves are the transvers magnetic waves of $3 \mathrm{GHz}$. The wide incident angle is divided into $1^{\circ}, 2^{\circ}, \ldots, 360^{\circ}$ in the first three examples and $0.1^{\circ}, 0.2^{\circ}, \ldots, 360^{\circ}$ in the last one. For the convenience of comparison, we define the recovery error as

$$
\Delta=\frac{\left\|\left[\widehat{\mathbf{I}}_{1} \widehat{\mathbf{I}}_{2}, \cdots, \widehat{\mathbf{I}}_{n}\right]-\left[\mathbf{I}_{1} \mathbf{I}_{2}, \cdots, \mathbf{I}_{n}\right]\right\|_{2}}{\left\|\left[\mathbf{I}_{1} \mathbf{I}_{2}, \cdots, \mathbf{I}_{n}\right]\right\|_{2}} .
$$

3.1. Example 1. An infinite perfectly electrical conducting (PEC) circular cylinder with a radius of $0.1 \mathrm{~m}$ is considered, and its generator is divided into 512 equally spaced segments. The impedance matrix and the excitation matrix are sparse in the wavelet domain after thresholding with the threshold of $10^{-4}$, as shown in Figures 1(a) and 1(b) (there are 128 nonzero rows in the excitation matrix). Figure 1(c) shows the matrix constructed by extracting 128 rows from the impedance matrix according to the indexes of nonzero rows in the excitation matrix.

The relationships between the number of measurements (i.e., the number of new sources $M^{\prime}$ ) and the recovery error are provided in Figure 2. It is clear that a lower number of measurements is required in the proposed scheme to achieve a similar precision as the conventional CS scheme.

The number of measurements is set to 35 , and only 64 rows in the projection $\Gamma$ need to be recovered since there are 64 nonzero rows in the measurement results of the rows of the current matrix $\left[\widetilde{\mathbf{I}}_{1} \widetilde{\mathbf{I}}_{2}, \cdots, \widetilde{\mathbf{I}}_{n}\right]$ (i.e., the solution $\widehat{\boldsymbol{s}}$ of (15)), as shown in Figure 3(a). The distribution of nonzero rows in the current matrix is shown in Figure 3(b). One can see clearly that the indexes of nonzero rows in the measurement results and the current matrix are the same. Hence, the prior knowledge provided by the measurement results is reliable. As shown in Figure 4, the numerical result of the original induced currents at the 77th segment under different incident angles is calculated by the proposed scheme, which also agrees very well with the result of traditional MoM.

3.2. Example 2. An infinite PEC square cylinder with a side length of $0.2 \mathrm{~m}$, whose perimeter is divided into 512 equally spaced segments, is taken as the object. The comparison of 


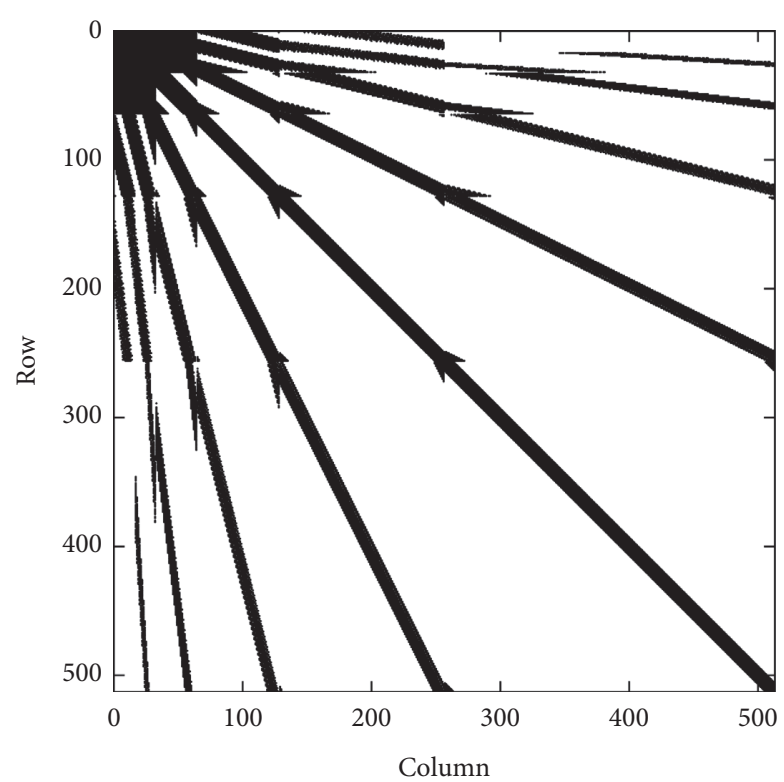

(a)

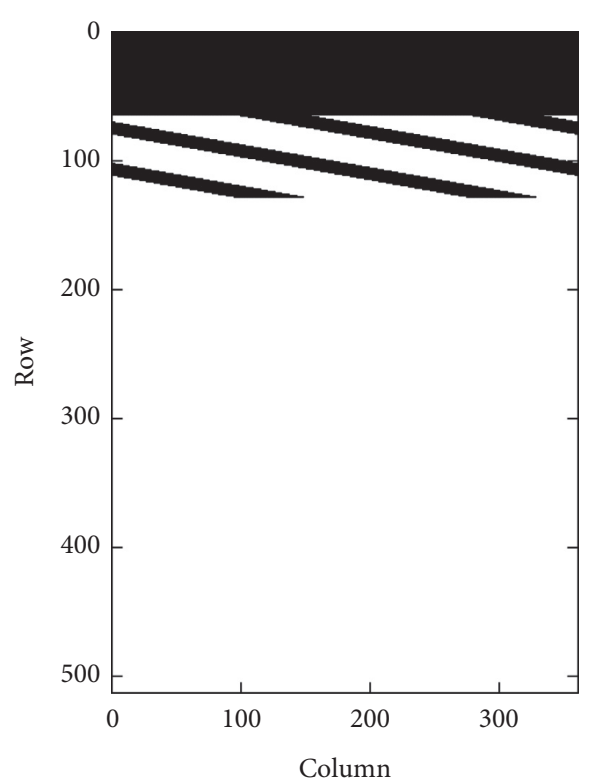

(b)

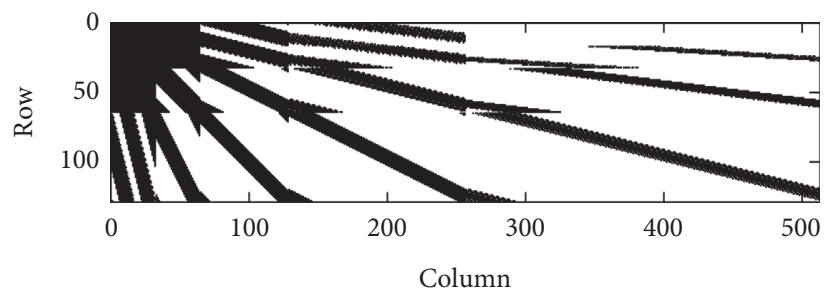

(c)

FIgURE 1: Matrices in wavelet domain. (a) Impedance matrix. (b) Excitation matrix. (c) Constructed matrix by extracting rows from the impedance matrix.

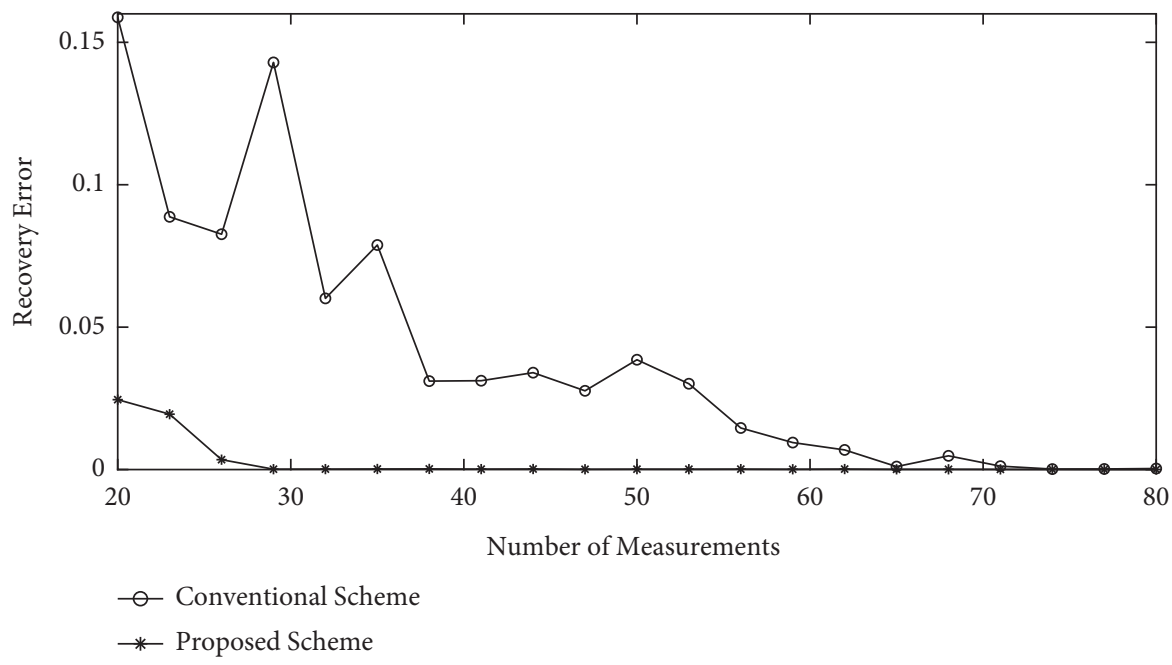

FIgURE 2: The recovery errors vary with the number of measurements for the circular cylinder.

measurement times between the conventional CS scheme and the proposed scheme is presented in Figure 5. It can be seen that only 39 measurements are needed to obtain the satisfactory accuracy by using the bilateral sparse transform in the proposed scheme, while the conventional CS scheme needs 78 calculations.
With the threshold of $10^{-3}, 184$ rows are extracted from the impedance matrix according to the indexes of nonzero rows in the excitation matrix. The number of rows that needs to be recovered in $\Gamma$ is only 128 with the help of the prior knowledge, which is shown in Figure 6(a) (the number of measurements is selected as 45). Figure 6(b) illustrates the distribution of nonzero 


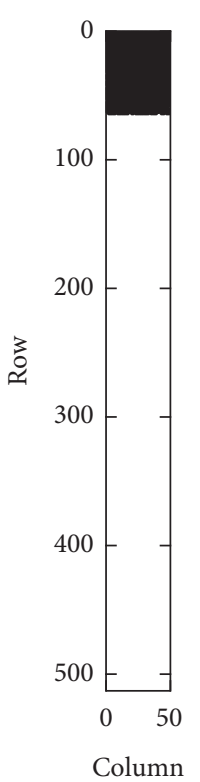

(a)

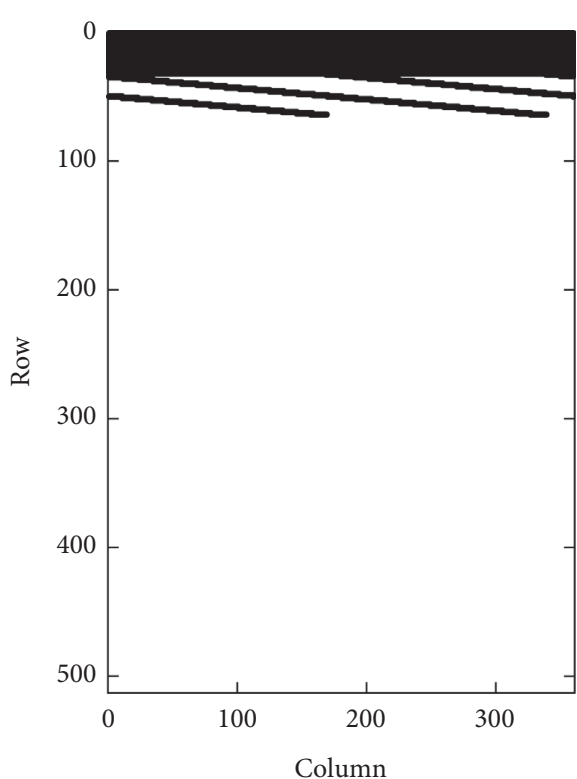

(b)

FiguRe 3: Distributions of nonzero rows in (a) measurement result matrix and (b) current matrix for the circular cylinder.

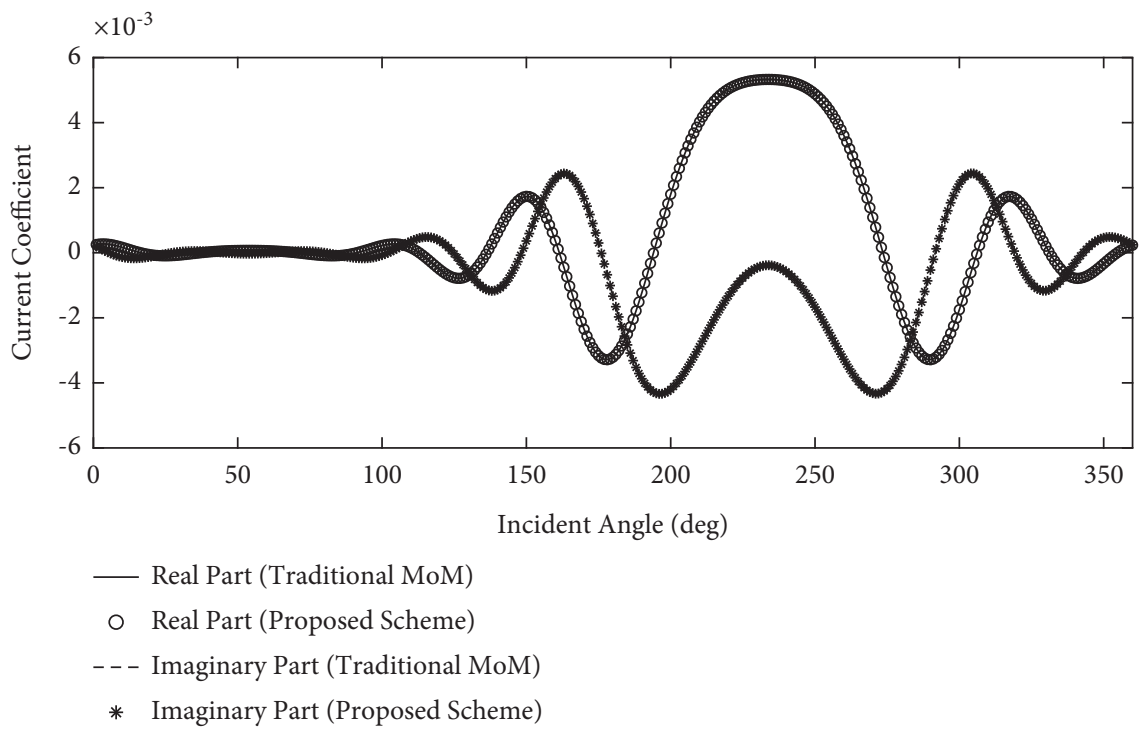

FIGURE 4: Calculation result of currents at the 77th segment of circular cylinder under different incident angles.

rows in the current matrix, and we can see that the indexes of nonzero rows in Figures 6(a) and 6(b) are identical. In Figure 7, the original induced current distribution at different segments with the incident angle at $70^{\circ}$ is presented, and the solution of traditional MoM is also provided for comparison. We can see that the proposed scheme has a high precision.

3.3. Example 3. As the third example, an infinite PEC concave cylinder (as shown in Figure 8) with 1024 basis functions is calculated. As shown in Figure 9, the comparison of the number of measurements proves the superiority of the bilateral sparse transform again. Setting the threshold and the measurement times to $10^{-3}$ and 65 , respectively, there are 360 nonzero rows in the excitation matrix, and thus, we need to extract the corresponding 360 rows from the impedance matrix. The distributions of nonzero rows in the measurement result matrix and the current matrix are shown in Figure 10. As can be seen, the distributions are still the same when the object is irregular. Therefore, only 250 rows in the projection need to be recovered. To verify the accuracy of the proposed scheme, the radar cross section (RCS) of the object illuminated by a random incident angle (e.g., $128^{\circ}$ ) is presented in Figure 11. 


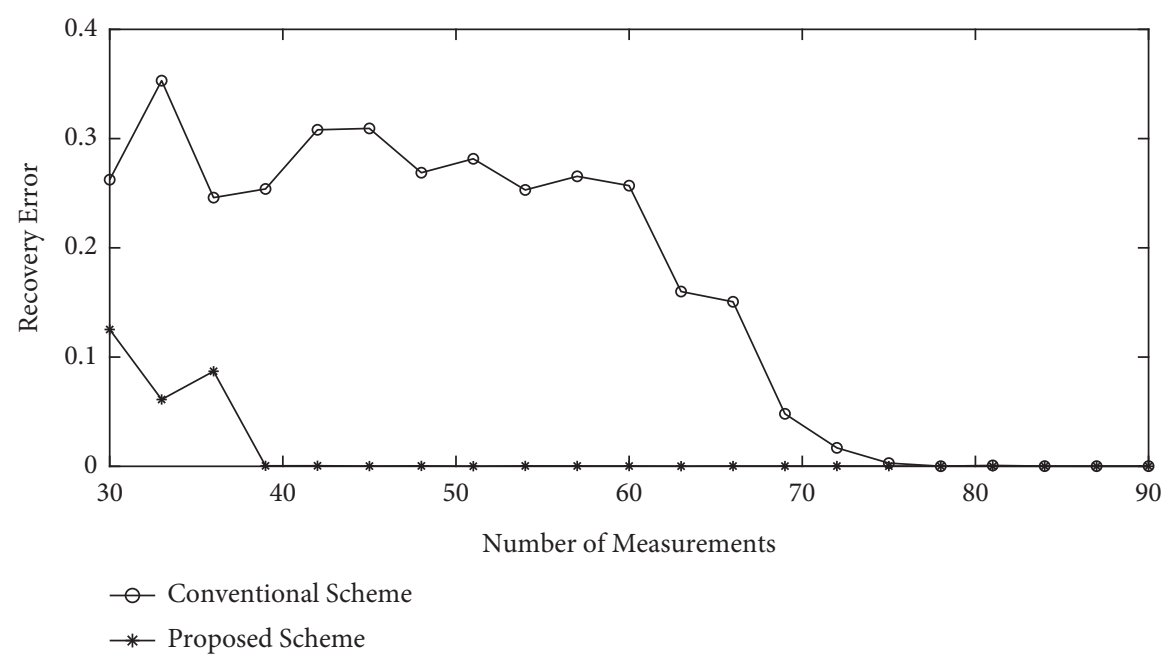

Figure 5: The recovery errors vary with the number of measurements for the square cylinder.

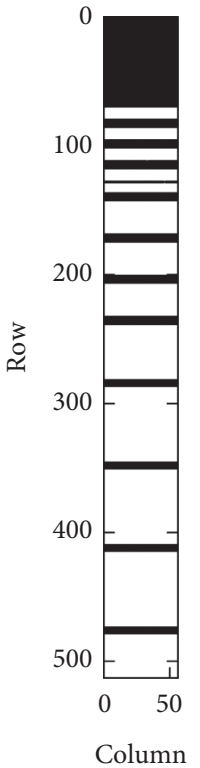

(a)

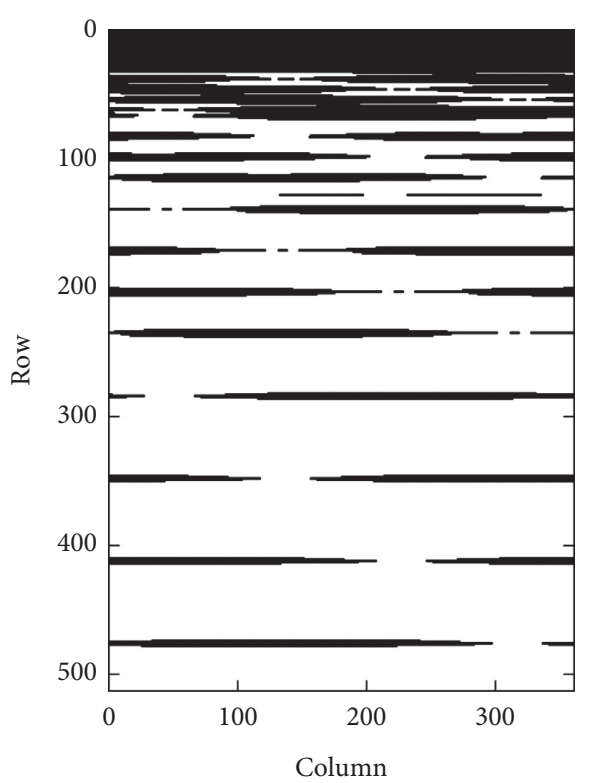

(b)

Figure 6: Distributions of nonzero rows in (a) measurement result matrix and (b) current matrix for the square cylinder.

3.4. Example 4. To prove the effectiveness of the proposed scheme for analyzing the electrically large targets, an infinite PEC circular cylinder with a radius of $10 \mathrm{~m}$ that contains 16384 basis functions is considered. We choose the threshold and the measurement times as $10^{-3}$ and 950, respectively, and 6121 rows are extracted from the impedance matrix to construct the measurement matrix to the columns in the current matrix. At last, 2353 nonzero rows in the current matrix need to be solved from (16). The RCS of the electrically large cylinder illuminated by a random incident angle (e.g., $77.7^{\circ}$ ) is shown in Figure 12, and the comparisons of CPU time and measurement times are provided in Table 1. 


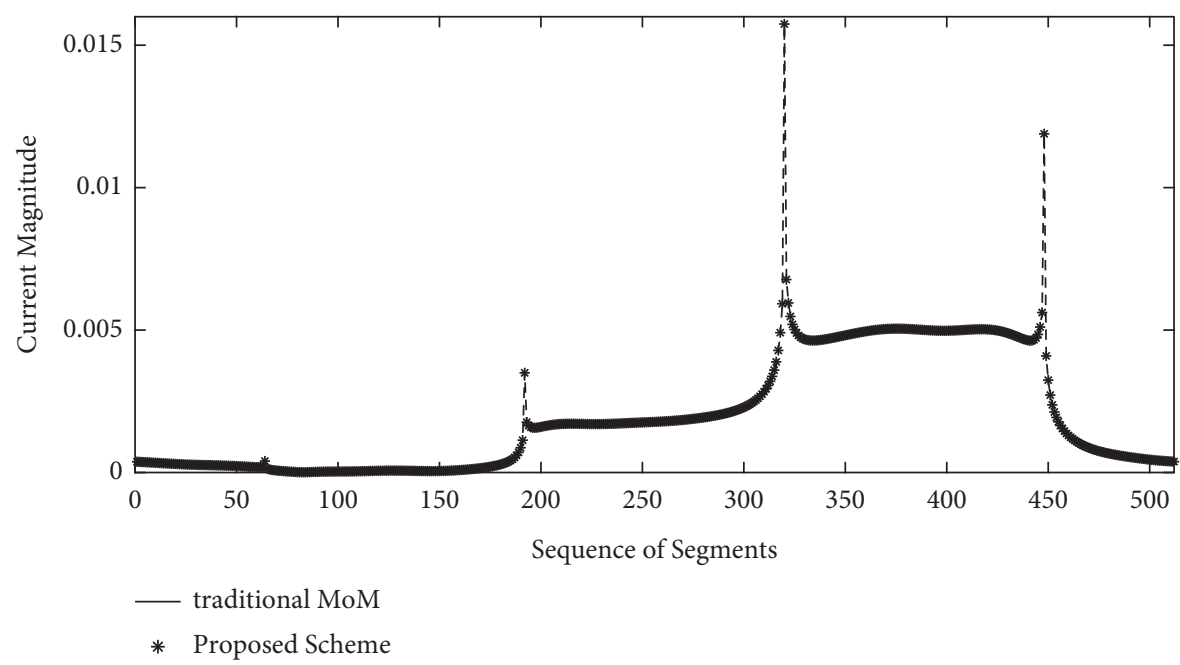

Figure 7: Current distribution of PEC square cylinder at different segments under a fixed incident angle at $70^{\circ}$.

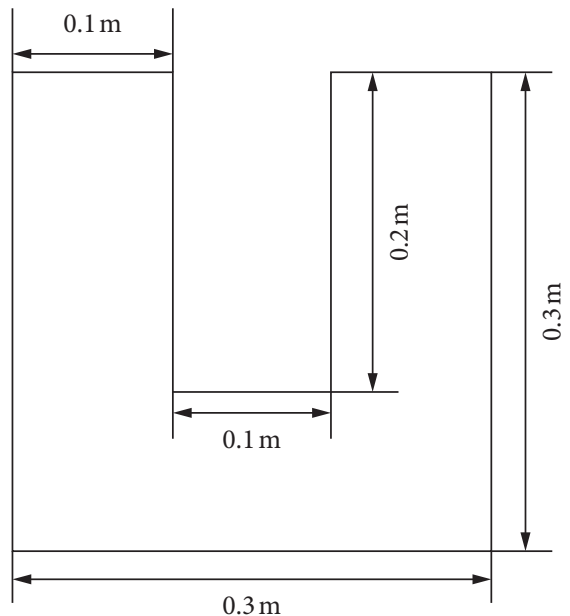

FIgURE 8: Cross section of the concave cylinder.

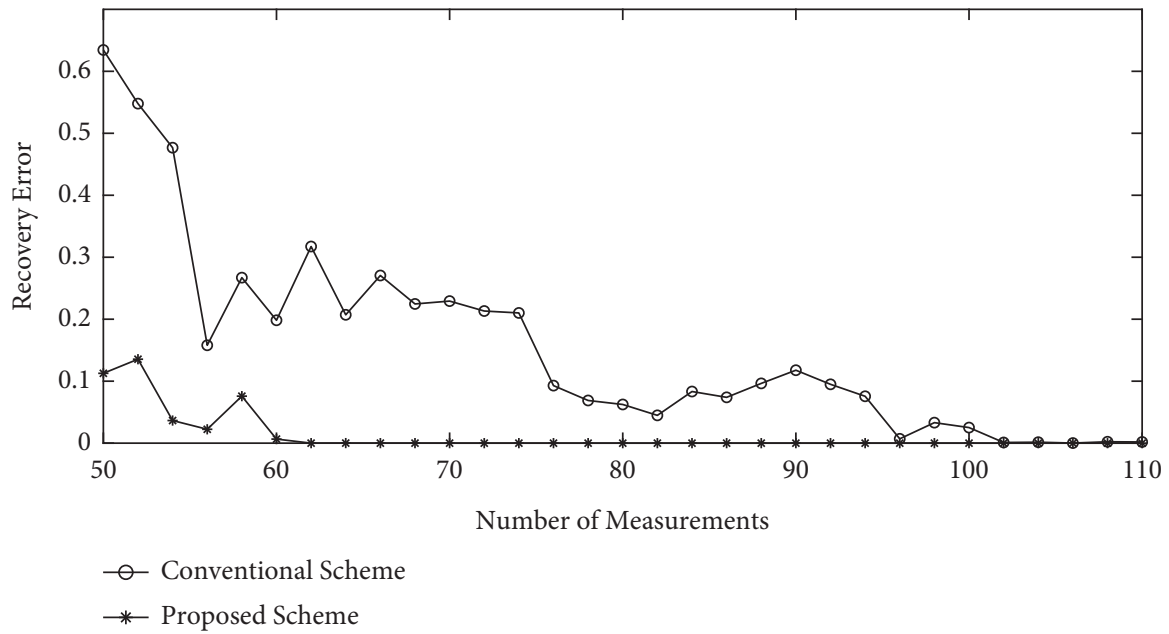

Figure 9: The recovery errors vary with the number of measurements for the concave cylinder. 


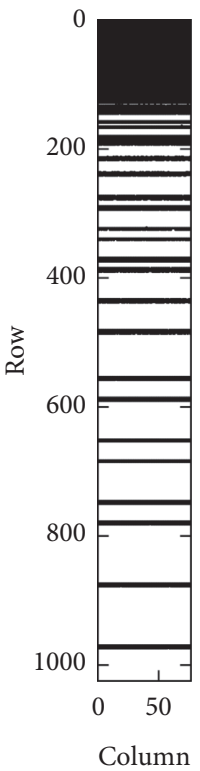

(a)

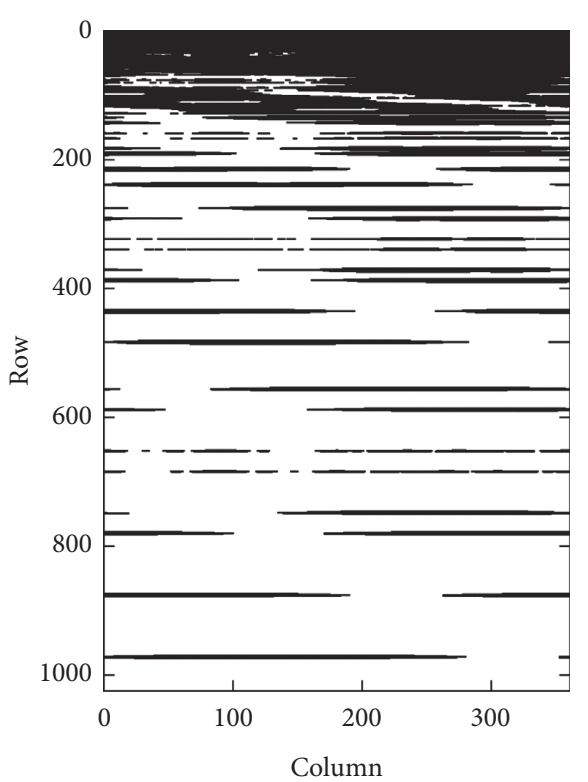

(b)

FIGURE 10: Distributions of nonzero rows in (a) measurement result matrix and (b) current matrix for the concave cylinder.

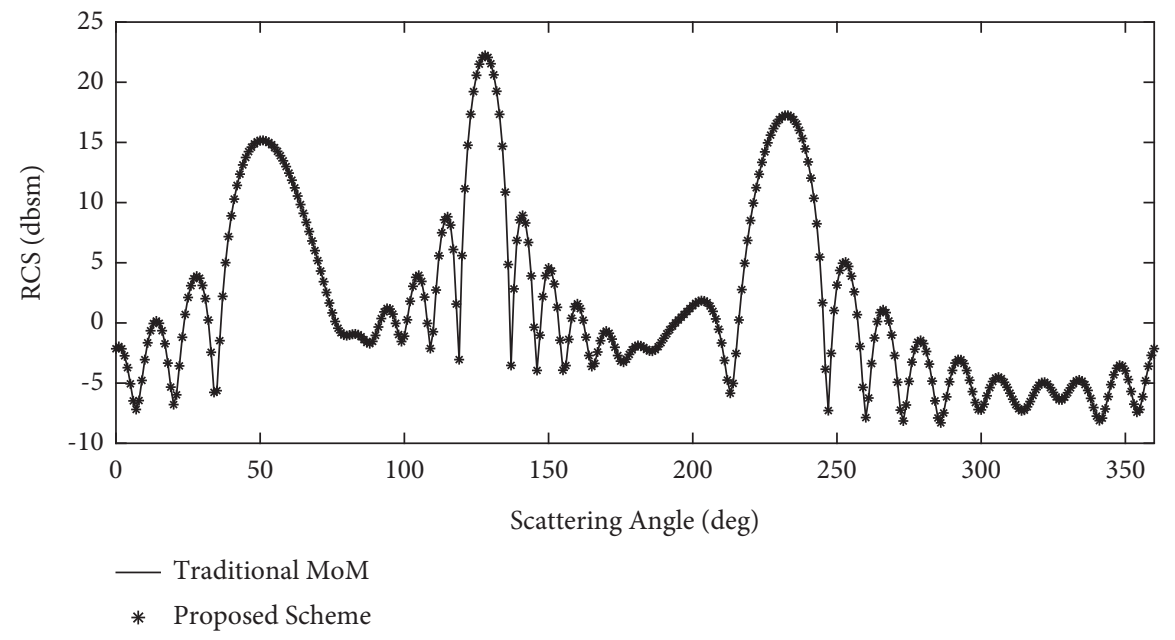

FIgURE 11: Comparison of RCS for the concave cylinder illuminated with an incident angle at $128^{\circ}$.

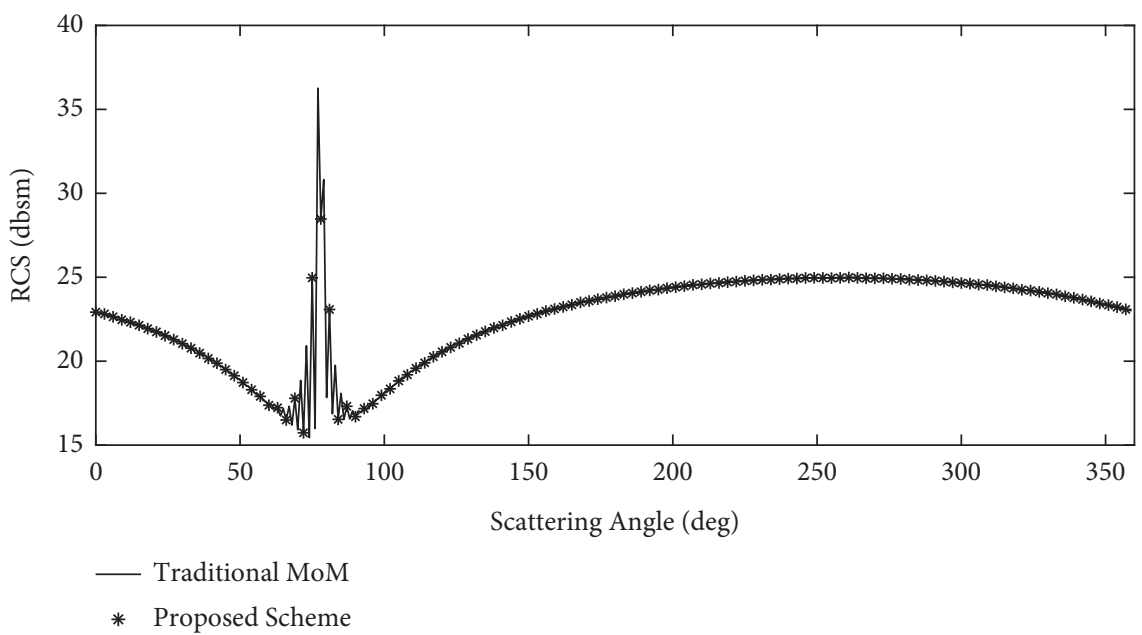

Figure 12: Comparison of RCS for the electrical cylinder illuminated with an incident angle at $77.7^{\circ}$. 
TABLE 1: Comparisons of CPU time and measurement times.

\begin{tabular}{lcc}
\hline Method & Measurement times & CPU time $(\mathrm{sec})$ \\
\hline Traditional MoM & - & 108521.8 \\
Conventional scheme & 1250 & 51004.7 \\
Proposed scheme & 950 & 15215.5 \\
\hline
\end{tabular}

\section{Conclusions}

In this paper, a 2-D CS scheme based on wavelet MoM is formed, by which the wide-angle scattering problems can be analyzed more efficiently. Numerical results demonstrate that only part of the rows in the projection matrix needs to be recovered with the help of the prior knowledge; meanwhile, compared with the conventional CS scheme, a lower number of measurements is required to obtain high accuracy in the proposed scheme.

\section{Data Availability}

The data used to support the findings of this study are available from the corresponding author upon request.

\section{Conflicts of Interest}

The authors declare that there are no conflicts of interest regarding the publication of this paper.

\section{Acknowledgments}

This work was supported by the National Natural Science Foundation of China under Grant nos. 61701163 and 61801163, the Anhui Provincial Key Research and Development Plan of China under Grant no. 201904a05020061, the Anhui Provincial Natural Science Foundation of China under Grant nos. 1808085MF167 and 1908085QF257, the Key Project of Provincial Natural Science Research of University of Anhui Province of China under Grant nos. KJ2019A0715 and KJ2020A0102, the Program for Excellent Youth Talents in Anhui Provincial Universities under Grant no. gxyqZD2019063, the Program of Anhui Province Key Laboratory of Simulation and Design for Electronic Information System under Grant no. 2019ZDSYSZY01, the Science and Technology Major Program of Anhui Province of China under Grant no. 201903a05020041, the Joint fund of Science \& Technology Department of Liaoning Province and State Key Laboratory of Robotics of China under Grant no. 2020-KF-22-16.

\section{References}

[1] R. F. Harrington, Field Computation by Moment Methods, IEEE Press, New York, NY, USA, 1993.

[2] J. Song, C. C. Lu, and W. C. Chew, "Multilevel fast multipole algorithm for electromagnetic scattering by large complex objects," IEEE Transactions on Antennas and Propagation, vol. 45, no. 10, pp. 1488-1493, 1997.

[3] W.-B. Ewe, L.-W. Li, C.-S. Chang, and J.-P. Xu, "AIM analysis of scattering and radiation by arbitrary surface-wire configurations," IEEE Transactions on Antennas and Propagation, vol. 55, no. 1, pp. 162-166, 2007.

[4] X. Chen, C. Gu, J. Ding, Z. Li, and Z. Niu, "Multilevel fast adaptive cross-approximation algorithm with characteristic basis functions," IEEE Transactions on Antennas and Propagation, vol. 63, no. 9, pp. 3994-4002, 2015.

[5] Q. Zhou, Y. Peng, and W. Wang, "On the use of wavelet MoM to solve the EFIE," Journal of Systems Engineering and Electronics, vol. 8, no. 4, pp. 23-33, 1997.

[6] C. J. Reddy, M. D. Deshpande, C. R. Cockrell, and F. B. Beck, "Fast RCS computation over a frequency band using method of moments in conjunction with asymptotic waveform evaluation technique," IEEE Transactions on Antennas and Propagation, vol. 46, no. 8, pp. 1229-1233, 1998.

[7] R. Baraniuk, "Compressive sensing [lecture notes]," IEEE Signal Processing Magazine, vol. 24, no. 4, pp. 118-121, 2007.

[8] S.-R. Chai and L.-X. Guo, "Compressive sensing for monostatic scattering from 3-D NURBS geometries," IEEE Transactions on Antennas and Propagation, vol. 64, no. 8, pp. 3545-3553, 2016.

[9] W. Zhu, M. Jiang, X. He, and J. Hu, "Compressive sensing based multilevel fast multipole acceleration for fast scattering center extraction and ISAR imaging," Sensors, vol. 18, no. 7, p. 2024, 2018.

[10] Q. Qi, X. Cao, M. Chen et al., “An improved fast finite element time-domain method based on compressive sensing for cavity problems," IEEE Microwave and Wireless Components Letters, vol. 30, no. 4, pp. 331-334, 2020.

[11] X. Cao, M. Chen, X. Wu, M. Kong, J. Hu, and Y. Zhu, "Dual compressed sensing method for solving electromagnetic scattering problems by method of moments," IEEE Antennas and Wireless Propagation Letters, vol. 17, no. 2, pp. 267-270, 2018.

[12] M. S. Chen, F. L. Liu, H. M. Du, and X. L. Wu, "Compressive sensing for fast analysis of wide-angle monostatic scattering problems," IEEE Antennas and Wireless Propagation Letters, vol. 10, pp. 1243-1246, 2011.

[13] S. Zhang, W. Zhang, Z. Zong, Z. Tian, and T. S. Yeo, "Highresolution bistatic ISAR imaging based on two-dimensional compressed sensing," IEEE Transactions on Antennas and Propagation, vol. 63, no. 5, pp. 2098-2111, 2015.

[14] J. A. Tropp and A. C. Gilbert, "Signal recovery from random measurements via orthogonal matching pursuit," IEEE Transactions on Information Theory, vol. 53, no. 12, pp. 4655-4666, 2007.

[15] R. L. Wagner and W. C. Chew, "A study of wavelets for the solution of electromagnetic integral equations," IEEE Transactions on Antennas and Propagation, vol. 43, no. 8, pp. 802-810, 1995.

[16] G. G. Pan, M. Jin, L. Zhang, M. Bai, and J. Miao, "An efficient scattering algorithm for smooth and sharp surfaces: coifletbased scalar MFIE," IEEE Transactions on Antennas and Propagation, vol. 62, no. 8, pp. 4241-4250, 2014.

[17] R. Baraniuk, M. Davenport, R. Devore, and M. Wakin, "A Simple proof of the restricted isometry property for random matrices," Constructive Approximation, vol. 28, no. 2, pp. 253-263, 2008.

[18] M. Kong, M. S. Chen, X. L. Wu, and B. Wu, "Fast and stabilized algorithm for analyzing electromagnetic scattering problems of bodies of revolution by compressive sensing," IEEE Antennas and Wireless Propagation Letters, vol. 16, pp. 198-201, 2016. 